\title{
A Construction Method of Semantic Weapon System of Systems Based on Ontology LI Kang ${ }^{a}$, LIU Dong ${ }^{b}$
}

(Equipment Academy, Beijing, 101416)

${ }^{a}$ E-mail: xtlikang1987@126.com, b E-mail: Id4m@139.com

Keywords: semantic; ontology; equipment system.

\begin{abstract}
This paper drawing idea of the Semantic Web and ontology technology advantages, semantic model proposed architecture and equipment - equipment system semantics, semantic concepts and definitions given in the relevant equipment system, discusses the semantic structure of the framework and building equipment system steps to semantic description of the equipment system based on multi-view.
\end{abstract}

\section{Introduction}

Research on the key issues facing the main equipment system requirements analysis[1], architecture modeling[2], architecture description and assessment system optimization, including architectural modeling is crucial to ensure that a comprehensive and integrated system of interoperable equipment, is the basis for research system[3]. The main contents of the general structure of the model equipment system including the composition of elements, consisting of a hierarchy of elements and composition relationships between elements of the three aspects, based on demand from based architecture, based on multiple perspectives on a task or ability to be considered, However, with the global perspective, the system was better able to multi-angle, multi-level, multi-granularity description model based architecture.

Currently, the relevant research equipment[4] [5], architecture modeling, mainly in the design and research model describing the conceptual level, there is no entity level architecture model equipped with the support from the equipment, which will result in the construction of the model and the actual application of the derailment. In this paper, based on the data architecture model building and equipment of ideas, through the existing real data mapping equipment systems and equipment for solid elements, building equipment available entity Collection, equipment specification describes various forms of entities, making interrelated organic whole. However, extensive real equipment data sources, with the structure of diverse types of complex semantic inconsistencies features that will directly mapped to the system, you can only get isolated from each other, a collection of meaningless equipment entity.

This paper drawing idea of the Semantic Web and ontology technology advantages, semantic model proposed architecture equipment - equipment system semantics (Semantic Weapon System of Systems-of-System, SemDAF), given the semantics of concepts and definitions related equipment system, discusses the semantic equipment structural frame and build steps, given the semantic description of the equipment system based on multi-view system.

\section{Semantically related concepts and equipment systems}

Semantics concept originally came from semantics, mainly refers to words in different contexts conditions have meaning. In the computer field, the semantic representation of the data represented by the meaning of the data in the different information that is represented in the information system. For example, one of the 18 digits "ID number" of data that can be presented Birthplace cities, counties, and date of birth and other information from different applications. Semantic field has obvious characteristics, how the data from different sources of organic association in semantic level up, the goal is the study of semantics. Here are semantically related equipment system semantic definitions: 
The role of the equipment according to its own performance characteristics play in specific equipment systems: semantic definition

1. Equipment (Equipment Semantic).

2. Definition of semantic systems and equipment (Equipment System-of-System Semantic): relationship constituent elements of the equipment system contained in a particular perspective and between.

Semantic (Capability Semantic) 3. Definition of equipment system capabilities: equipment system complete a specific task in a specific context condition skills.

Ontology (Ontology) originally comes from the philosophy, used to describe the thing itself, after being introduced to the field of artificial intelligence, used to describe the basic concepts and relationships within a certain field, and said that the rules of these concepts and relationships. Body language can be used to provide a clear specification of a shared conceptualization of things in the form of abstract modeling shows that to achieve the semantic level between heterogeneous systems sharing and interoperability. Depending on the application level of the body, can be divided into field-level ontology (Domain Ontology), application-level ontology (Application Ontology), data-level ontology (Data Ontology). Relationships within the domain ontology describes the basic concepts of the field definitions and concepts, the application of the body is under some kind of knowledge of a specific application mode data, data ontology is based on the concept of formal description description reasoning. Here are definitions of ontology semantic systems and equipment:

1. Definition of equipment body (Equipment Ontology): part of the data-level ontology, describing the specific parameters of information equipment entity.

2. Definition of the equipment system domain ontology (Equipment Domain Ontology): belongs to the field-level ontology, field equipment system is described in the basic concepts and relationships between concepts, defines the relationship between the type of equipment, property and equipment, equipment and equipment .

3. Definition of equipment system capacity body (Equipment System-of-System Capability Ontology): is an application-level ontology, describing the equipment system to complete a specific task in a specific background conditions skills, the ability to define the metric type, capacity, capability and capacity relationships.

Semantic Weapon System of Systems is based on real-world equipment architecture model has been constructed equipment, computer equipment entity in the world in the form of data records exist, each data record corresponds to a well-equipped entity, the entity will be equipped by the semantic integration of data processing, convert it to equip the body of interrelated, so that all the equipment into a unified organic whole in the system, which constitutes the Semantic Weapon System of Systems.

Compared with the existing methods and equipment system modeling, Semantic Weapon System of Systems has two main advantages: First, the equipment based on real data, the actual image of the data through the establishment of systems and equipment for research equipment system to provide high-quality data support; the second is based on semantic description ontology, can use a unified language to describe the various elements of the system equipment, eliminating the problem of heterogeneous and heterogeneous semantic grammar within the system, to achieve interoperability and integration of equipment, systems and infrastructure to provide semantic grammar specification.

\section{Ontology semantic systems and equipment}

Equipment System process includes mission requirements analysis, system capacity planning, operational elements generation, integrated use of equipment, etc., this section describes the ontology-based combat system, process equipment, first of all, from the task needs, clear goals and battlefield information; secondly, by decomposing the task, planning to complete the system's ability to combat missions; then extracted to meet the conditions of combat equipment, systems and equipment to generate operational elements; Finally, according to the performance characteristics of the planning battle plan and equipment, integrated use of a variety of equipment to complete combat 
missions.

This section is based RDF ontology language, namely the equipment body, the relationship between the body and equipment, the ability to describe the equipment system.

1. Ontology semantic description of equipment

Establish equipment number, name, type, tactical and technical indicators such as property and equipment body, unified by a unique identifier URI in the global scope, describing the parameters of the equipment body. Define the relationship between several main equipment: belonging to, deployed, equivalent to three semantic relations.

\begin{tabular}{|l|l|}
\hline describe & URI \\
\hline WeaponID & http://www.yyzbtx.com/Weapon/ID\# \\
\hline WeaponName & http://www.yyzbtx.com/Weapon/Name\# \\
\hline WeaponType & http://www.yyzbtx.com/Weapon/Type\# \\
\hline WeaponProperty & http://www.yyzbtx.com/Weapon/Property\# \\
\hline BelongTo & http://www.honhoo.com/relation/BelongTo\# \\
\hline LocatedIn & http://www.honhoo.com/relation/LocatedIn\# \\
\hline EquivalentTo & http://www.honhoo.com/relation/EquivalentTo \# \\
\hline
\end{tabular}

2. Task ontology requirements analysis

Operational scenario described in mission requirements, you can perform a series of operational activities to complete. Each operational activities executed by an instance of an Activity, Activity instance of the class with examples Rules and Weapon class description of the task execution time, the battlefield environment, the combat zone, operational objectives and other information, sample code given below.

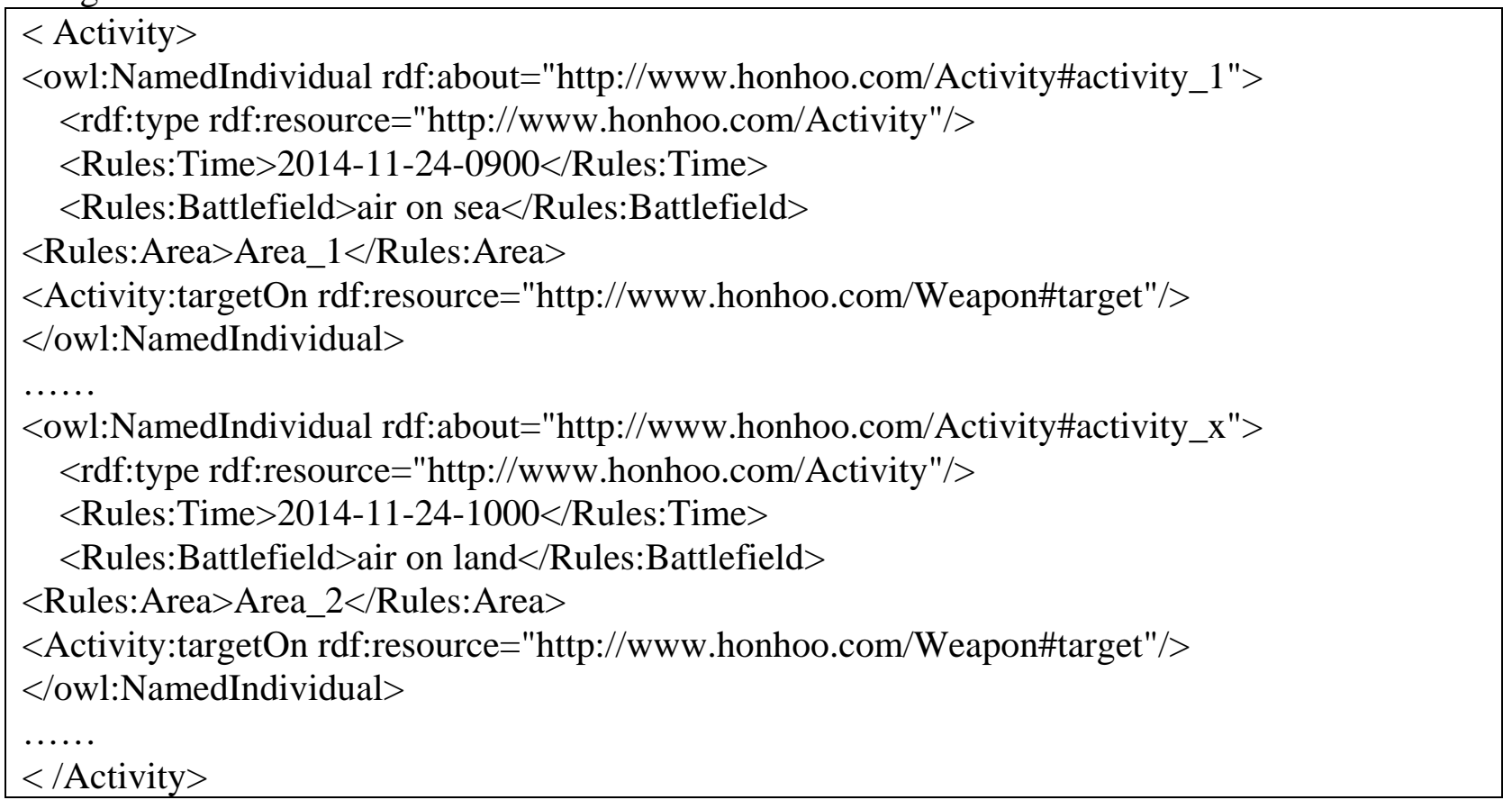

3. ontology system capacity planning

By analyzing the characteristics of mission requirements, the ability to type in accordance with the task decomposed into sub-tasks, and then through an instance CapPlan and Rules with examples classes and Capability class planning system capacity needs in different time periods, the following sample code is given . 


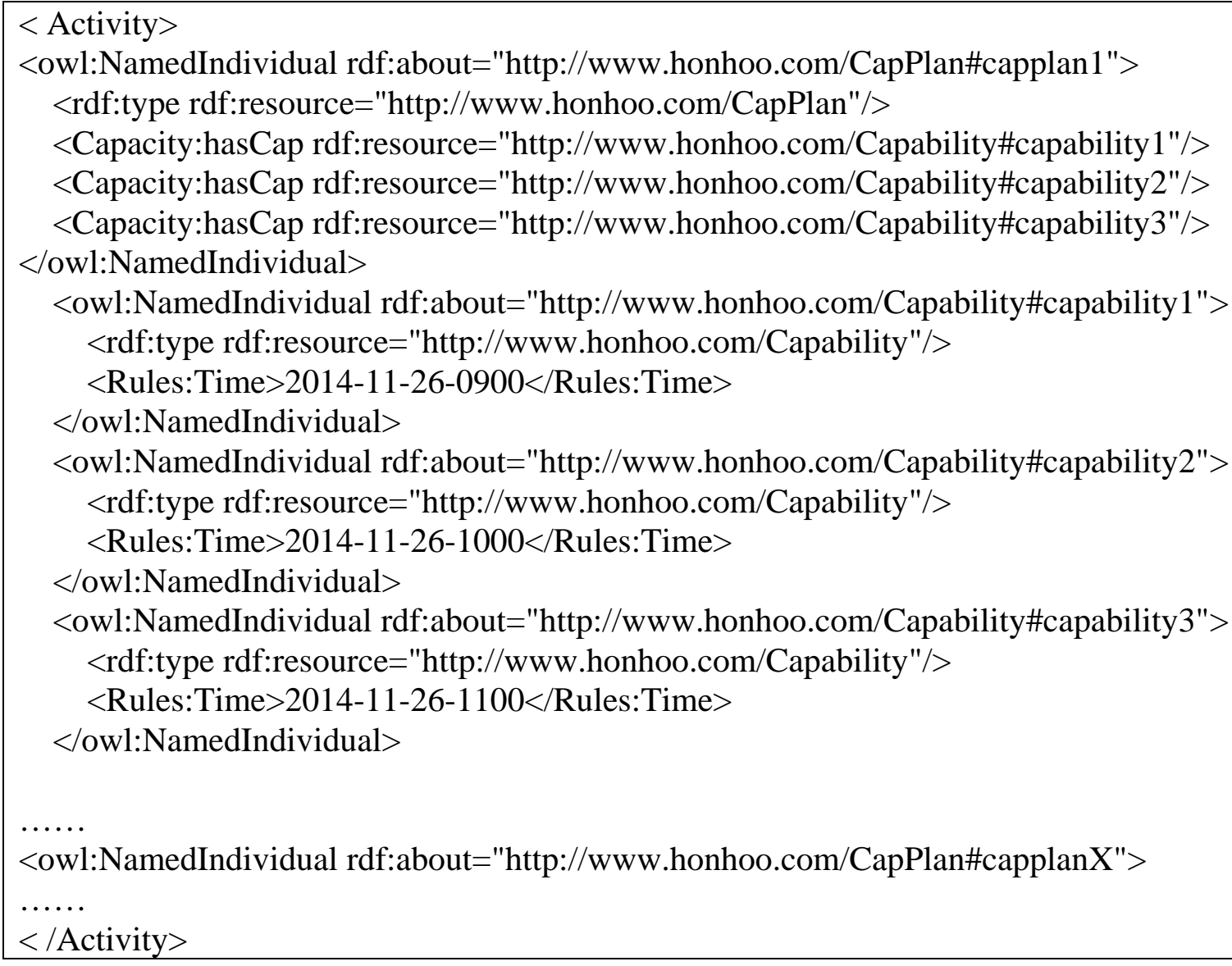

\section{Summary}

Equipment system is a complex giant system, its description of the structure is multifaceted. This paper drawing idea of the Semantic Web and ontology technology advantages, semantic model proposed architecture equipment - equipment system semantics, semantic concepts and definitions given in the relevant equipment system, discusses the semantic structure of the framework and building equipment system steps given on semantic description of the equipment system multi-view method.

\section{References}

[1] Zhaoqing Song, Yang Kewei, CHEN Ying-wu and other systems engineering and architecture modeling methods and techniques [J]. Beijing: National Defense Industry Press, 2013.

[2] Tour glory, beginning Jun Tian and other research on weaponry system [M] Military Operations Research and Systems Engineering, 2010, 24 (4): 15-22.

[3] Gebing Feng, CHEN Ying-wu, Wang Junmin and other weapons and equipment based on the structure of the system functions described method [J] Systems Engineering and Electronics, 2010, 32 (1): 94-99.

[4] Armament architecture is based on the mission and methodological framework for modeling Ye Guoqing, Yu Shu, etc. [J] Fire Control and Command Control, 2012, 37 (7): 1-5.

[5] Tan Yuejin, Zhang can, Yang Kewei weaponry system network description and modeling methods [J] System Management Journal, 2012, 21 (6): 781-786. 